\title{
Modelización de las inestabilidades en la deformación plástica de monocristales de circona estabilizada con itria (YCSZ)
}

\author{
A. GALLARDO-LÓPEZ, D. GÓMEZ-GARCíA, A. DOMÍNGUEZ-RODRÍGUEZ Y L. KUBIN \\ Departamento de Física de la Materia Condensada-Universidad de Sevilla \\ Apartado 1065-4180, Sevilla, España \\ ${ }^{+}$LEM, CNRS/ONERA \\ 29 Av. de la Division Leclerc, BP 72, 92322 Châtillon Cedex, France
}

\begin{abstract}
En este trabajo se presenta un modelo para explicar las inestabilidades dinámicas observadas durante la deformación plástica a alta temperatura de monocristales de circona estabilizada con itria, (YCSZ, yttria cubic stabilized zirconia) con alto contenido en itria. El modelo se basa en el fenómeno Portevin-Le Chatelier (PLC), que consiste en episodios sucesivos de anclaje y desanclaje de dislocaciones cuando éstas se mueven con una velocidad del mismo orden que los defectos que obstaculizan su movimiento. La evaluación numérica de los parámetros implicados en el modelo muestra que las inestabilidades plásticas son totalmente compatibles con el efecto Portevin- Le Chatelier.
\end{abstract}

Palabras clave: YCSZ, monocristal, propiedades mecánicas, inestabilidades plásticas, efecto PLC, modelización

\section{Modelling of instabilities in the plastic deformation of yttria stabilized zirconia single crystals}

In this work, a model explaining dynamic instabilities observed during high temperature plastic deformation of single crystalline yttria cubic stabilized zirconia (YCSZ) is presented. The model is based on the Portevin-Le Chatelier phenomenon, which consists of pinning and unpinning of dislocations when they move with a velocity of the same order of magnitude than the defects which act as obstacles to their movement. Numerical evaluation of the parameters implied in the model shows that plastic instabilities are fully compatible with a Portevin-Le Chatelier effect.

Keywords: YCSZ, single crystal, mechanical properties, plastic instabilities, Portevin-Le Chatelier effect, modelization.

\section{INTRODUCCIÓN}

El estudio de las propiedades mecánicas a alta temperatura de la circona monocristalina dopada con itria en fase cúbica (YCSZ) adquiere especial relevancia, por cuanto éstas dan información de propiedades básicas esenciales que son de ineludible conocimiento para el diseño de cerámicas avanzadas que contienen como componente a la circona. Los monocristales de circona itriada con contenido en itria por encima del $9 \mathrm{~mol} \%$ (fase cúbica) presentan un endurecimiento por solución sólida a medida que aumenta la concentración de itria, alcanzando un máximo para la concentración de $21 \mathrm{~mol} \%$ (1-3). Sin embargo, se han observado inestabilidades durante la deformación plástica del material en condiciones muy concretas de velocidad de deformación, tensión y temperatura, en muestras con alto contenido en itria, que vienen siendo objeto de estudio en los últimos años. El comportamiento plástico inestable para concentraciones superiores al $18 \mathrm{~mol} \%$ de itria fue observado por primera vez por McClellan et al (4) quienes propusieron un mecanismo de tipo térmico (calentamiento y ablandamiento localizado de bandas de deslizamiento) para explicarlo. Estudios posteriores (5-6) pusieron de manifiesto mediante experimentos especialmente diseñados, que las características del fenómeno se correspondían más bien con un mecanismo de tipo Portevin-Le Chatelier (PLC) que consiste en episodios alternativos de anclaje y desanclaje de dislocaciones cuando la velocidad de los defectos que obstaculizan su movimiento y la velocidad de deslizamiento de las propias dislocaciones son del mismo orden de magnitud.
En este trabajo se propone un modelo teórico en el que se estiman y se comparan los tiempos característicos de vuelo y espera de las dislocaciones entre los obstáculos y los tiempos de difusión de dichos obstáculos. El que estos tiempos sean del mismo orden de magnitud indicaría que la existencia de un mecanismo Portevin-Le Chatelier está justificada en este sentido.

\section{PARTE EXPERIMENTAL}

El material objeto de este estudio es la circona itriada cúbica monocristalina (YCSZ) con 21, 24, 28 y $32 \mathrm{~mol} \%$ de contenido en itria. Se han llevado a cabo experimentos de compresión uniaxial con el eje de compresión en la dirección cristalográfica [1 12$]$ para activar el sistema de deslizamiento primario $[\overline{1} 0]_{001)}$. En estos ensayos ha sido crucial el control de la temperatura y la velocidad de deformación para propiciar la aparición del comportamiento inestable. Los experimentos se realizaron a distintas temperaturas en el intervalo 1310$1450^{\circ} \mathrm{C}$ y a velocidades de deformación entre $1,6 \cdot 10^{-5} \mathrm{y}$ $3,2 \cdot 10^{-4} \mathrm{~s}^{-1}$. También se han realizado observaciones mediante microscopía electrónica de transmisión de la subestructura de dislocaciones de las muestras deformadas. Esto nos ha permitido obtener datos experimentales de parámetros como la densidad de dislocaciones móviles, que luego utilizaremos en el modelo. 


\section{RESULTADOS Y DISCUSIÓN}

Tanto los ensayos mecánicos como las observaciones microestructurales coinciden al indicar que las inestabilidades plásticas se producen en los primeros estadios de la deformación plástica, lo cual corresponde a la activación del plano de deslizamiento primario [1 10$](001)$, ya que las muestras estaban orientadas con su eje mayor (eje de compresión) en la dirección cristalográfica $[1 \overline{1} 2]$ (de "deslizamiento fácil"). La micrografía de la figura 1 muestra el plano (001) de deslizamiento primario con una densidad de dislocaciones $\rho \approx$ $10^{13} \mathrm{~m}^{-2}$. Las dislocaciones son preferentemente rectas, en arista y con vector de Burgers en la dirección [ $[\overline{1} 0]$, como es típico en YCSZ. Se observa igualmente la formación de bucles y dipolos característicos de una interacción dislocación-defectos puntuales, condición para que se produzcan inestabilidades dinámicas.

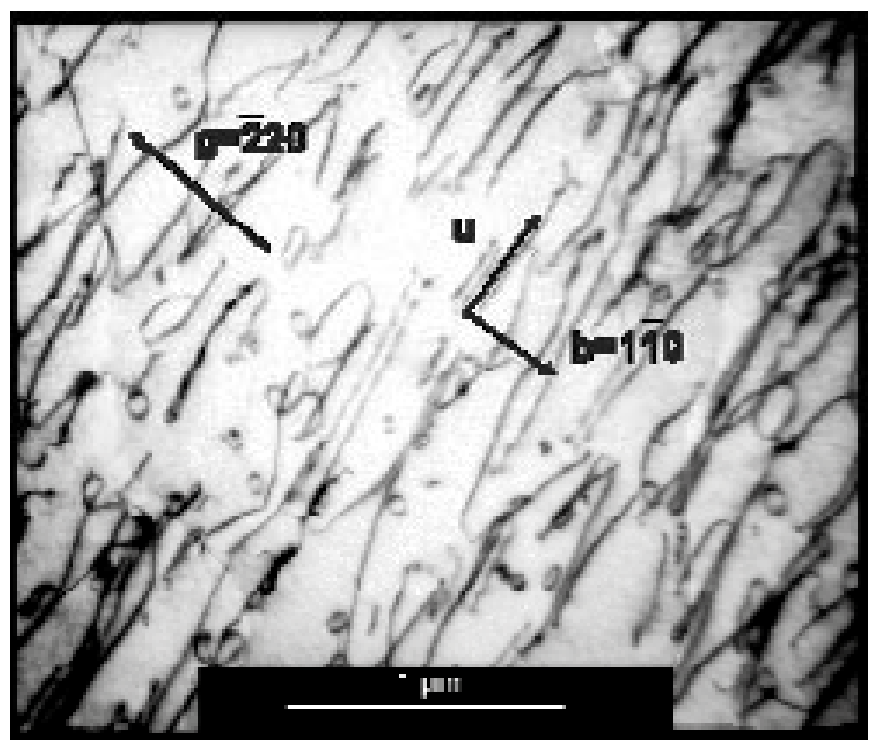

Fig. 1- Subestructura de dislocaciones en el plano de deslizamiento primario (001) de una muestra con $24 \mathrm{~mol} \% \mathrm{Y}_{2} \mathrm{O}_{3}$ deformada hasta el $3 \%$ en la que se observaron inestabilidades dinámicas. Se indican el vector $\vec{g}$, el vector de Burgers de las dislocaciones, $\vec{b}$, y la línea de dislocación $\vec{u}$. La mayoría de las dislocaciones están en arista. La densidad media de dislocaciones se ha estimado en $\rho=10^{13} \mathrm{~m}^{-2}$.

Los resultados de ensayos mecánicos de estudios previos delimitan un rango finito de velocidades de deformación y de temperatura en los que tiene lugar la deformación inestable, lo cual es reflejo de las condiciones para las cuales la velocidad con la que los defectos difunden hacia las dislocaciones es comparable con la velocidad de deslizamiento de las propias dislocaciones. Otros datos experimentales apuntan también en esta misma dirección. La caída abrupta de la sensibilidad a la velocidad de deformación en el rango inestable es característica de fenómenos de envejecimiento dinámico (7). También aparece una dependencia anómala de la tensión con la temperatura en el intervalo estudiado, (es decir, la tensión permanece casi constante al aumentar la temperatura, ver figura 2), que puede deberse al aumento de la cantidad de soluto que ancla las dislocaciones al aumentar la temperatura. Experimentos de envejecimiento estático muestran también que existe una especie que difunde hacia las dislocaciones (ver figura 2). Cada uno de estos hechos tomados de forma aislada no sería suficiente para caracterizar un efecto Portevin-
Le Chatelier. Sin embargo, la aparición conjunta de todos estos fenómenos puede ser considerada como una prueba fehaciente.

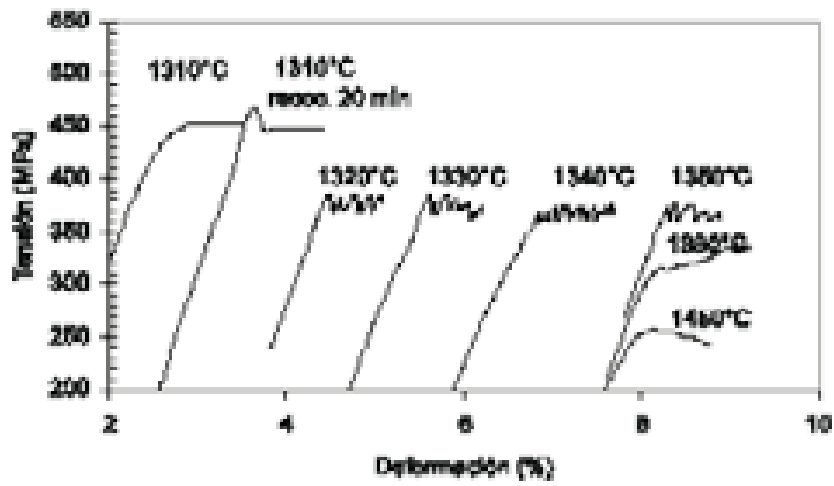

Fig. 2- Curvas tension-deformación correspondientes a muestras con $24 \mathrm{~mol} \% \mathrm{Y}_{2} \mathrm{O}_{3}$, deformadas plásticamente en compresión uniaxial tal y como se describe en la parte experimental, a una velocidad de deformación de $\dot{\varepsilon}=1.610^{-5} \mathrm{~s}^{-1}$ y entre 1310 y $1450^{\circ} \mathrm{C}$ como se indica. Las curvas correspondientes a $1310^{\circ} \mathrm{C}$ corresponden a un experimento de envejecimiento estático (obsérvense los picos de tensión).

En YCSZ con alto contenido en itria, el mecanismo que controla la tensión de límite elástico y la de fluencia en condiciones de deslizamiento fácil es la interacción de las dislocaciones con defectos complejos. Estos defectos están formados por la asociación de dos átomos de itrio y una vacante de oxígeno (8). Por tanto, sería razonable pensar que las especies que difunden y provocan las inestabilidades plásticas sean átomos sustitucionales de itrio. Podemos verificar esta hipótesis comprobando que, en las condiciones en las que se observa la deformación inestable, la movilidad de los átomos de itrio por difusión es del mismo orden que la movilidad de las dislocaciones. En particular, podemos ver si el tiempo típico de difusión durante el cual los átomos de soluto difunden hacia el corazón de las dislocaciones, $\tau$, es del mismo orden que el tiempo efectivo que emplean las dislocaciones en saltar de un obstáculo al siguiente junto con el tiempo de espera, $t$. Para ello usaremos un cálculo numérico basado en el modelo típico para envejecimiento dinámico por deformación del efecto PLC (Portevin le-Chatelier) (9). Cálculos similares se han llevado a cabo con éxito para aleaciones de aluminio con átomos de soluto de metales de transición, permitiendo identificar las especies de soluto que difundían (10).

Las condiciones óptimas para que se produzcan inestabilidades tipo PLC son:

$$
\mathrm{t}_{\mathrm{w}} \approx \tau
$$

En primer lugar debemos estimar el tiempo de espera para las dislocaciones, que es el tiempo durante el cual permanecen ancladas. Cuando se liberan, se mueven hacia el siguiente obstáculo durante un tiempo de vuelo despreciable. Si llamamos c a la concentración de defectos de itrio, cada defecto ocupa $\mathrm{c} / \mathrm{a}^{2}$ sitios atómicos por unidad de área del plano de deslizamiento, donde a es el parámetro reticular. La distancia promedio entre obstáculos es entonces $\Lambda=a / \sqrt{c}$. La velocidad promedio entre obstáculos es: $\bar{v}=\Lambda / t_{w}=a /\left(t_{w} \sqrt{c}\right)$ , es decir, el cociente entre el recorrido libre medio y el tiempo que tarda una dislocación en superar un obstáculo y llegar al siguiente (en la práctica el tiempo de espera, $t_{w}$ ). La 
velocidad de deformación plástica, $\dot{\varepsilon}$, viene dada por la ley de Orowan:

$$
\dot{\varepsilon}=\rho_{m} b \bar{v}=\frac{\rho_{m} b a}{t_{w} \sqrt{c}}
$$

donde $\rho_{\mathrm{m}}$ es la densidad de dislocaciones móviles y b es el modulo de su vector de Burgers. Para YCSZ, $\mathrm{b}=0.36 \mathrm{~nm}$, a $=0.5 \mathrm{~nm}$ y la concentración de defectos de itrio es $\mathrm{c}=0.15$ (11). Como la mayoría de las dislocaciones son móviles en condiciones de deslizamiento simple, tomamos $\rho_{\mathrm{m}}=10^{13} \mathrm{~m}^{-2}$ (ver Fig. 1). Con una velocidad de deformación impuesta $\dot{\varepsilon}$ $=1.6 \cdot 10^{-5} s^{-1}$, la ecuación (2) da un tiempo de espera $\mathrm{t}_{\mathrm{w}} \approx$ $0.49 \mathrm{~s}$. Este valor, que es del orden de $1 \mathrm{~s}$, es un valor típico para el PLC en aleaciones metálicas (9-10).

Podemos estimar ahora el tiempo de difusión de los átomos de itrio, $\tau$. Un cálculo clásico de difusión (12) nos lleva a:

$$
\tau=\frac{\mathrm{k}_{\mathrm{B}} \mathrm{Tb}^{2}}{\mathrm{n}(\mathrm{n}+2) \mathrm{DW}_{\mathrm{M}}}\left(\frac{\mathrm{c}_{1}}{\pi \mathrm{c}_{0}}\right)^{\frac{\mathrm{n}+2}{2}}
$$

En esta expresión, $\mathrm{n}$ es un entero que depende de la naturaleza del mecanismo de interacción entre los átomos de soluto y las dislocaciones. Aquí, hemos tomado $\mathrm{n}=1$, valor típico cuando se tienen en cuenta los efectos de talla (las tensiones generadas por el mayor radio iónico de los defectos sustitucionales de itrio en comparación con los átomos de circonio). $\mathrm{D}$ es el coeficiente de difusion de los átomos de itrio en YCSZ, que ha sido determinado como $\mathrm{D}=\mathrm{D}_{\mathrm{o}} \exp (-$ $\left.\mathrm{Q} / \mathrm{k}_{\mathrm{B}} \mathrm{T}\right)$, donde $\mathrm{k}_{\mathrm{B}}$ es la constante de Boltzmann. De acuerdo con (13), $\mathrm{D}_{\mathrm{o}}=4,8210^{-6} \mathrm{~m}^{2} \mathrm{~s}^{-1}$ y la energía de activación para la difusión en volumen de los átomos de itrio es $\mathrm{Q}=4,86$ $\mathrm{eV}$. Una temperatura clásica para el efecto PLC en estos experimentos es $\mathrm{T} \approx 1640 \mathrm{~K}$, con lo cual $\mathrm{k}_{\mathrm{B}} \mathrm{T} \approx 0,144 \mathrm{eV}$. Como resultado, tenemos $\mathrm{D}=1,1110^{-20} \mathrm{~m}^{2} \mathrm{~s}^{-1}$. $W_{M}$ es la energía de interacción de los átomos de soluto de itrio con el corazón de las dislocaciones, que ha sido estimada como $\mathrm{W}_{\mathrm{M}} \geq 1 \mathrm{eV}$. (12). $\mathrm{c}_{\mathrm{o}}$ es la concentración atómica de soluto que difunde y en las condiciones experimentales $\mathrm{c}_{0} \approx 0,14 . \mathrm{c}_{1}$ es la concentración máxima de soluto en las dislocaciones ancladas. Como este valor es menor que el de saturación, $\mathrm{c}_{\mathrm{s}}=1$, tomamos $\mathrm{c}_{1} \approx 0,5$ tal como indica Friedel (12).

Con los datos numéricos dados anteriormente, obtenemos $\tau=0,68 \mathrm{~s}$, que es comparable al valor hallado para el tiempo de espera $t_{w}=0,49 \mathrm{~s}$. Por tanto, aunque algunas de las constantes físicas utilizadas en estos cálculos no son conocidas con gran precisión, el resultado final indica que el itrio es, con alta probabilidad, la especie de soluto responsable del efecto PLC en el sistema $\mathrm{Y}_{2} \mathrm{O}_{3}-\mathrm{ZrO}_{2}$.

\section{CONCLUSIONES}

Se ha utilizado un modelo típico para envejecimiento dinámico por deformación del efecto PLC junto con datos empíricos obtenidos en experimentos de deformación en compresión uniaxial para estimar el tiempo que tarda una dislocación en superar un obstáculo (tiempo de espera) y el tiempo de difusión de los obstáculos complejos de itrio hasta las dislocaciones. La comparación de estos tiempos indica que son del mismo orden de magnitud, lo cual está en acuerdo con que el fenómeno Portevin- Le Chatelier, que involucra el anclaje y desanclaje de dislocaciones por obstáculos sea el causante de las inestabilidades plásticas observadas en YCSZ con alto contenido en itria durante su deformación por deslizamiento simple a alta temperatura.

\section{AGRADECIMIENTOS}

Este trabajo ha sido financiado por los proyectos de colaboración hispano-francesa HF2001-0125 y HF2003-0272.

\section{BIBLIOGRAFÍA}

1.D. Gómez-García, J. Martínez-Fernández, A. Domínguez-Rodríguez, J. Castaing. "Mechanisms of high-temperature creep of fully stabilized zirconia single crystals as a function of the yttria content". J. Am. Ceram. Soc., 80 (7), pp. 1668-72, 1997.

2. D. Gómez-García, J. Martínez-Fernández, A. Domínguez-Rodríguez , K.H. Westmacott. "Zirconium Nitride Precipitation in Nominally Pure YttriaStabilized Zirconia“. J. Am. Ceram. Soc., 79 [2], pp. 487-490, 1996.

3.A. Gallardo-López, J. Martínez-Fernández, D. Gómez-García, A. DomínguezRodríguez, "High Temperature Plastic Deformation of 24-32 mol\% Yttria Cubic Stabilized Zirconia (YCSZ) Single Crystals". J. Eur. Ceram. Soc., vol. 23/13 pp. 2183-2191, 2003.

4. K.J. McClellan, A.H. Heuer, L.P. Kubin. "Localized yielding during high temperature deformation of $\mathrm{Y}_{2} \mathrm{O}_{3}$-fully-stabilized cubic $\mathrm{ZrO}_{2}$ single crystals". Acta Metal., 44, [7], pp. 2651-2662, 1996

5. A.Gallardo-López, D. Gómez-García, A. Domínguez-Rodríguez, L.P. Kubin. "Portevin-Le Chatelier effect in $\mathrm{Y}_{2} \mathrm{O}_{3}-\mathrm{ZrO}_{2}$ single crystals". Scripta Mater., 51 pp. 203-207, 2004.

6. A.Gallardo-López, D. Gómez-García, A. Domínguez-Rodríguez, L.P. Kubin. "Inestabilidades dinámicas en cerámicas monocristalinas a base de circona", Bol. Soc. Esp. Ceram. V., 44, [2], pp. 95-101, 2005.

7. A.H.Cottrell. Dislocations and plastic flow in crystals. London. Oxford University Press, p. 147, 1953.

8. F.R. Chien and A.H.Heuer. "Lattice diffusion kinetics in $\mathrm{Y}_{2} \mathrm{O}_{3}$-stabilized cubic $\mathrm{ZrO} 2$ single crystals: a dislocation loop annealing study". Phil. Mag. A;73, 3, pp. 681-697, 1996.

9. Y. Estrin, and L.P. Kubin. "Plastic instabilities: clasification and physical mechanisms". Res Mechanica, 23, pp. 197-221, 1998.

10. E. Bouchaud, L. Kubin and H. Octor. "Ductility and dynamic strain aging in rapidly solidified aluminum alloys". Metall. Trans., 22A, p.1021, 1991.

11. D. Gómez-García, A. Gallardo-López , J. Martínez-Fernández, A. Domínguez-Rodríguez. "Influence of defects on the high temperature mechanical behaviour of yttria fully-stabilized zirconia single crystals". Rad. Eff. Def. Sol., 151-11, p. 895, 1999.

12. J. Friedel. Dislocations. Pergamon Press , pp. 368-84, 405, 1964.

13. M. Kilo, G. Borchardt, S. Weber, S. Scherrer and K. Tinschert. "Zirconium and calcium tracer diffusion in stabilized cubic zirconia". Ver Bunsenges Phys Chem., 101, p.1361, 1997.

Recibido: 09.01.05

Aceptado: 14.03 .05 